\title{
POLEMICAS
}




\section{ENTUSIASMO Y DESILUSION DE UN PROGRAMA DE EDUCACION A DISTANCIA POR TELEVISION: \\ EL CASO DEL FONDO DE CAPACITACION POPULAR*}

Milciades Vizcaíno G. $^{* * *}$ Julio Ernesto Díaz $B^{* * *}$

\section{"Capacitación popular: Palanca del progreso"}

Este logo comenzó a aparecer en los hogares colombianos, en el Canal 11 de sus televisores, el 13 de noviembre de 1968. El 26 de febrero de $1970^{10}$, Carlos Lleras Restrepo, Presidente de la República, inauguró el nuevo sistema de educación a distancia con una "teleclase" de civismo. El Presidente grabó su intervención en el Palacio de San Carlos y, en una forma dialogada, comenzó a dictar su conferencia.

El hecho de que el Presidente de la República inaugurara el canal de T.V. y fuera el primero en dictar una clase por el canal 11, el cual sería dedicado exclusivamente a la capacitación y educación de adultos, reflejaba el compromiso que el gobierno buscaba asumir con el programa.

En 1982 el presidente Belisario Betancur incluye dentro de su programa de gobierno un plan ambicioso de educación a distancia. Por ello resulta conveniente revisar los logros y fracasos del mayor experimento oficial que en este campo se ha realizado en el país.

En este artículo se describirá el programa educativo de Capacitación Popular por televisión; se analizará cómo fueron elaborados los cursos; qué papel jugó la investigación en el desarrollo de los mismos; y por último, cuál es la situación actual de los programas y de la institución responsable de la realización de los mismos.

\section{La educación de adultos y los medios de comunicación}

La revolución cubana abre la década de los sesenta. Este acontecimiento obligó a los países latinoamericanos y a los Estados Unidos a replantear sus políticas tanto económicas como sociales. Para dicho propósito, se reúnen en Punta del Este (Uruguay) los jefes de Estado de los países americanos en agosto de $1961^{11}$.

Uno de los acuerdos centrales que allí se produjo se refería a la ampliación de la educación para erradicar el analfabetismo y proporcionar educación básica a los sectores marginados. La educación de los adultos tendría un papel prioritario para los gobiernos ${ }^{12}$.

\footnotetext{
* Las enseñanzas del Dr. Azriel Bibliowicz fueron definitivas para la realización de este trabajo. A él un reconocimiento de parte de los autores.

** Sociólogo. M.A. en Investigación Socioeducativa, profesor de la Universidad Cooperativa INDESCO y funcionario del Fondo de Capacitación Popular.

${ }^{* * * *}$ Sociólogo. M.A. en Investigación Socioeducativa y profesor de la Universidad del Valle.

${ }^{10}$ Entre los meses de noviembre de 1968 y febrero de 1970 el canal se usó para múltiples pruebas y ensayos hasta que por último fue inaugurado oficialmente por el Presidente con su "teleclase". En 1970 quedaron completadas las instalaciones técnicas que permitieron que el canal cubriera a los departamentos de Cundinamarca, Tolima, Caldas (5 municipios), Antioquia (8 municipios) y Huila (2 municipios).

${ }^{11}$ La comisión colombiana que preparó los documentos que sirvieron de base para la elaboración del Plan Decenal de Educación incluido en la Carta de la Alianza para el Progreso estaba integrada por: Guillermo Nanchi, Gabriel Betancur M., Jaime Posada, Luis Córdoba M., Gabriel Anzola G. y Antonio Díaz. (Ver. LEBOT, lvon. "Elementos para la historia de la educación en Colombia en el siglo XX". Bogotá, Departamento Administrativo Nacional de Estadistica (DANE), mayo de 1978, p. 159.

${ }^{12}$ Se esperaba alfabetizar en América Latina a 48 millones de adultos, dentro de los acuerdos de la Alianza para el Progreso. (Ver: LEBOT, lvon. Ibídem). 
Con el fin de garantizar la ejecución de esta política de educación masiva se impulsó la utilización de los medios de comunicación en la educación.

Los medios de comunicación de masas, debido a su difusión y penetración, se vieron como la panacea para el desarrollo de estos programas educativos. No fueron pocos los estudios que enfatizaban sus ventajas a nivel de costo-beneficio, así como sus cualidades para acabar con el analfabetismo en el continente ${ }^{13}$.

Los flujos migratorios campo-ciudad generaban múltiples problemas de carácter social. Cinturones de miseria, tugurios, aparecieron en todas las grandes ciudades del continente. Esta compleja situación fue analizada por los expertos, en los famosos estudios de la época sobre la "marginalidad"14.

Se afirmaba que no era posible emprender actividades de beneficio social ni productividad económica mientras la población no estuviera alfabetizada. La educación se constituía en la llave maestra que daría el salto del desarrollo a los países subdesarrollados ${ }^{15}$.

La incorporación de mano de obra a la producción era un aspecto preocupante. La mano de obra disponible, en particular la migrante, no tenía la preparación necesaria y suficiente para sumir la operación de máquinas y herramientas sofisticadas. Requerían de la educación para que ésta les diera por lo menos los elementos básicos para poder leer unas instrucciones, y la empresa se encargaría, salvo otra alternativa, de la preparación específica en el campo de trabajo.

Por otra parte, la migración campo-ciudad se veía como uno de los problemas que limitaban tanto la aplicación de las políticas del Estado como los procesos económicos, especialmente con el desempleo creciente. El presidente Lleras Restrepo en aquellos días planteaba que: "las proporciones de la migración superan las posibilidades de crear empleos razonables remunerados en la industria y en los servicios. Además de esto, hay que añadir que parte de los migrantes tienen bajo nivel cultural y poca o ninguna preparación para los oficios urbanos"16.

El impulso inicial que tuvo el Fondo de Capacitación Popular tiene una relación directa con los planteamientos esbozados en el Plan de la Transformación Nacional del presidente Lleras Restrepo y con una política para preparar e integrar estas poblaciones marginadas al desarrollo industrial.

\section{Capacitación Popular: Planes ambiciosos y realizaciones pobres.}

\footnotetext{
${ }^{13}$ Un estudio de Tiffin afirmaba que "en la década del sesenta la televisión educativa era encarada como una solución técnica revolucionaria para los gigantescos problemas educacionales de América Latina". TIFFIN, John. "Problemas críticos en sistemas de Televisión Educativa", en Tecnología Educativa. Santiago de Chile, No. 2, Vol. 4, 1978. p. 164. También ver: GORDON, George N. Televisión Educativa. México: UTEHA, 1966.

${ }^{14}$ Ver por ejemplo: DESAL. Marginalidad en Américo Latina. Santiago de Chile: DESAL-HERDER, 1969. VEKEMANS, Roger y otros. Marginalidad, Promoción Popular e Integración Latíinoarnericana. Santiago de Chile: DESAL y Buenos Aires: Troquel, 1970. GERMANI, Gino. El concepto de Marginalidad. Buenos Aires: Nueva Visión, 1973.

${ }^{15}$ SOLARI, Aldo. "Desarrollo y política educacional en América Latina", en Revista de la CEPAL, Santiago de Chile, Primer semestre de 1977, p. 61-94.

${ }^{16}$ LLERAS RESTREPO, Carlos. "Mensaje a los Directorios de los Partidos Políticos, en EL TIEMPO. Bogotá, mayo 29 de 1969. 2 a. sección.
} 


\section{La capacitación popular en el marco de la política internacional}

Cuando se inició Capacitación Popular en 1967, sus fundadores estaban convencidos que habían creado un modelo de educación de adultos para América Latina.

En un artículo sobre el tema, José Galat, consejero presidencial de la administración Lleras Restrepo y uno de los artífices de la idea, presentó el proyecto de la siguiente forma: "Capacitación Popular, el programa más audaz que se realiza en Colombia utilizando las técnicas modernas de los medios de comunicación, en concreto la radio y la televisión "17.

El proyecto se proponía adelantar la más gigantesca campaña para llevar educación masiva y en forma acelerada a los sectores populares de la población colombiana ${ }^{18}$.

Por medio del sistema ${ }^{19}$ de Capacitación Popular se intentaría hacer un cubrimiento de la población que al final de la década del 60 no había tenido oportunidad de escolarizarse o que habiendo sido excluida del sistema regular de enseñanza, había olvidado por desuso lo aprendido. El consejero Galat calculaba que por lo menos dos terceras partes de los colombianos podrían ser beneficiarios potenciales del nuevo proyecto educacional $^{20}$.

Pero esta capacidad de cubrimiento, de masividad no dejaba de ser un sueño desproporcionado si se tenía en cuenta las condiciones precarias en que se inicia su funcionamiento.

Capacitación Popular comienza a tener vida como institución en mayo de 1967. Para esta época sólo 12 personas conformaban el equipo de trabajo. Seis pertenecían al proyecto y las demás eran voluntarias o venían en comisión de diferentes entidades del Estado $^{21}$.

La oficina donde comenzó a operar el programa era un local prestado por Colsubsidio, una empresa particular de subsidio familiar. Los sueldos de los empleados eran cubiertos con aportes financieros de la Empresa de Licores de Cundinamarca y de tres entidades bancarias $^{22}$.

\footnotetext{
${ }^{17}$ GALAT NOUMER, José. “Colombia un gigantesco Centro Educativo”. Bogotá: Revista Javeriana, No. 358, septiembre de 1969. Pág. 298.

${ }^{18}$ En 1969 el Gobierno colombiano sostenía que buscaba alfabetizar a dos millones de colombianos de las ciudades, con el programa de Capacitación Popular. (Galat Noumer, José 1.c). El Presidente Betancur, el 17 de septiembre de 1982, sostenía que la Campaña de Instrucción Nacional - CAMINA - va dirigida a cinco millones de colombianos (Ver: EL TIEMPO. Bogotá, 18 de septiembre de 1982).

${ }_{19}$ Debido a que la televisión en este caso se consideraba un "sistema suplementario" del maestro, la emisión no era general sino dirigida a unos telecentros. El canal buscaba ser un circuito cerrado ampliado; sin embargo, la emisión podía ser recibida en cualquier hogar colombiano. Si bien al comienzo se creyó que el uso de los medios de comunicación masiva por sí solos creaban un "sistema" a la hora de la verdad en ningún momento Capacitación Popular logró diferenciarse del sistema tradicional de educación que impera en el país. Se sacó al maestro autoritario del aula y se le puso en el televisor, sin hacer un manejo adecuado del medio.

${ }^{20}$ GALAT NOUMER, José. Op. Cit.

${ }^{21}$ El 1o. de mayo de 1967, la Oficina de la Consejería de la Presidencia de la República a cargo del José Galat, en desarrollo del Programa de Integración Popular, instaló una oficina provisional en un local cedido por la Caja Colombiana de Subsidio Familiar (Colsubsidio) con el siguiente personal: Pedro Arreaza, director; Alonso Moncada, sociólogo; Jesús Bailén, experto en educación de adultos del Ministerio de Educación Nacional; María Cristina Jiménez, dibujante; Helena Méndez, dibujante del SENA; Alfonso Téllez, dibujante de los Cuerpos de Paz; Adriano Torres, Publicista; Hilda de González, secretaria de Inravisión; Marlén Sanabria, mensajera; Diego Castillo, voluntario; Astrid Escobar, voluntaria. Ver (Pedro Arreaza, "Informe sobre las actividades desarrolladas por el Fondo de Capacitación Popular desde el mes de mayo de 1967 hasta el mes de octubre de 1969" Capacitación Popular. Bogotá: Octubre de 1969, p. 1).

${ }^{22}$ La Licorera de Cundinamarca aportó dos millones de pesos; el Banco Santander \$25 mil; la Caja Colombiana de Ahorros $\$ 150$ mil; y el Banco de Construcción y Desarrollo $\$ 35$ mil. (Ver: Pedro Arreaza, Ioc. cit.).
} 
En junio del mismo año el gobierno le dio vida jurídica a Capacitación Popular y a través de la junta directiva de Inravisión creó un "Fondo" en el que supuestamente depositarían sus aportes financieros las más diversas entidades públicas, privadas, nacionales e internacionales.

Este Fondo sería administrado por Inravisión pero dependería directamente del Consejero Presidencial que tenía bajo su responsabilidad el gran proyecto oficial de "integración Popular", del cual Capacitación Popular no era sino un aspecto "operativo"23.

Con el proyecto de "Integración Popular" el gobierno aspiraba a encontrar soluciones a los problemas de salud, vivienda, educación y cultura, empleo, recreación,.., etc., etc., de la población marginada ${ }^{24}$.

Dos años después, según el informe del primer Director, Capacitación Popular todavía no había encontrado las formas de resolver el problema financiero para cubrir al menos los gastos ordinarios de funcionamiento. Los aportes de las entidades oficiales y privadas no llegaron y aunque el Ministerio de Educación se había comprometido por medio de un convenio a ayudar a financiar el proyecto, los recursos no eran suficientes y además tardaban mucho tiempo debido a los trámites burocráticos ${ }^{25}$.

En estas condiciones Capacitación Popular no había logrado sostener mas de una hora diaria de programación televisiva y en la recepción de los programas tan sólo habían unos 50 telecentros en el oriente y sur de Bogota.

Robert Arnove, un sociólogo norteamericano que participó en algunas investigaciones sobre la efectividad de los programas de Capacitación Popular, hizo una apreciación del proyecto en esta primera época. “...el programa de Capacitación Popular no tuvo éxito por varias razones. Un currículo irreal e irrelevante, programas de televisión mediocres, monitores sin entrenamiento y sin motivación para trabajar por la comunidad, un calendario de clases poco realista, que duraba nueve meses y sobre todo la baja probabilidad de que los participantes mejoraran sus condiciones económicas y sociales, pronosticaban su fracaso" 26 .

Este comentario contrastaba dramáticamente con la apreciación ilusoria de quien fuera el primer director de Capacitación Popular.

\footnotetext{
${ }^{23}$ Acuerdo 09 del 8 de junio de 1967 por el cual la Junta Administradora de Inravisión constituye el Fondo de Capacitación Popular. El Presidente Carlos Lleras Restrepo afirmaba que la integración Popular no crearía nueva burocracia, sino que ante todo era "un mecanismo para hacer" (Charla televisada del 20 de septiembre de 1966 dirigida a todo el país por las dos cadenas comerciales de televisión y la Radio Nacional. Ver: Carlos Lleras Restrepo, Mensaje Presidencial. Bogotá: Editorial Andes, 1970, p. 33). Igual afirmación sobre que el nuevo programa no crearía una nueva burocracia lo han hecho el presidente Betancur y el ministro de Educación Dr. Jaime Arias Ramírez, en relación con la Campaña de Instrucción Nacional — CAMINA— con ocasión de la promulgación del Decreto 2412 sobre Educación Abierta y a Distancia. (Bogotá, septiembre de 1982).

${ }^{24}$ Decreto 2263 de 1966 por el cual el Gobierno estimula y organiza la Integración Popular. El proceso de industrialización de la década de los sesenta presentaba una exigencia a la educación: la Preparación del personal adecuado. El impulso de los Institutos Técnicos Agrícolas (ITAS) las escuelas agropecuarias, el Servicio Nacional de Aprendizaje (SENA) y los Institutos de Educación Media Diversificada (INEM) se presentaron como una solución educacional a los requerimientos del desarrollo industrial.

El Gobierno del Dr. Lleras esperaba que la misión de los organismos de integración popular radicaría en coordinar y movilizar todos los rodajes gubernamentales. (Charla televisada realizada el día 27 de octubre de 1966 por los dos canales de televisión, la Radio Nacional y emisoras particulares; en Carlos Lleras Restrepo, Mensaje Presidencial. loc. cit. p. 46.

${ }^{25}$ ARREAZA, Pedro. op. cit.

${ }^{26}$ ARNOVE, Robert: "Políticas educativas durante el Frente Nacional" en Revista Colombiana de Educación. No. 1, Primer Semestre de 1978. p. 37.
} 
En su informe de despedida de su primera administración aseguraba que: "la efectividad del programa había sido satisfactoria y el sistema podría convertirse en modelo para otros países" 27 .

\section{El Cuento Rosado de Julio y Rosa}

Con la entrada del segundo director de Capacitación Popular, René Hauzeur Forero, en 1970, la situación económica del Fondo mejoró sustancialmente con los aportes del Estado y de fundaciones y organismos internacionales, lo cual permitió que, la institución pudiera comenzar a cumplir con algunos planes iniciales ${ }^{28}$.

A pesar de que hubo un incremento considerable de aportes financieros de diferentes entidades, la capacidad operativa de la Institución distaba mucho de posibilitar la expansión de la red de televisión, de mejorar los equipos o de proyectar innovaciones significativas en la estructura del programa.

Para esta época el canal ya tenía fama de tener una programación muy aburrida. Numerosas críticas se hacían a la programación de alfabetización, no sólo desde el punto de vista técnico-pedagógico sino desde la perspectiva de los contenidos ideológicos que resultaban ser una completa desfiguración de la realidad.

Julio y Rosa, los personajes de este primer curso emitido iban y venían entre la perfección y la idiotez. Los usuarios preguntaban por qué extraña razón Julio y Rosa nunca se enfermaban, nunca peleaban, no tenían problemas de ningún tipo, trabajaban sin descanso y daban gracias a Dios por haberlos creado y haberles concedido la vida y la salud para seguir trabajando. Eran totalmente caricaturescos y algunos funcionarios de la Institución los catalogaban de "angelicales" ${ }^{29}$.

Por otra parte la recepción del programa era caótica. En los Telecentros se estaba presentando una deserción de más del $60 \%$ entre los estudiantes que seguían las clases por televisión. Según la investigación que estudiaba este fenómeno, los usuarios no mostraban ningún interés por el programa y los guías eran incapaces de asesorarlos y de motivarlos en su proceso de aprendizaje ${ }^{30}$.

Una de las primeras decisiones importantes del segundo Director de Capacitación Popular René Hauzeur fue la de archivar definitivamente el programa de Julio y Rosa. La decisión se fundamentó en la presión de los usuarios y en el consejo de los expertos consultados $^{31}$.

\footnotetext{
${ }^{27}$ ARREAZA LLERAS, Pedro. Loc. cit. p. 9.

${ }^{28}$ Ver: ARREAZA, Pedro. loc. cit.; FONDO DE CAPACITACION POPULAR "El Fondo de Capacitación Popular". Bogotá. Octubre de 1971; FONDO DE CAPACITACION POPULAR. "Memorias sobre el Fondo de Capacitación Popular, 1967-1981". Bogotá, abril de 1981.

${ }^{29}$ Informes de los supervisores de los telecentros y de los investigadores. Ver Documentos: “Quién es Julio —y- Quién es Rosa. Fondo de Capacitación Popular. Bogotá, noviembre de 1970.

${ }^{30}$ Observaciones de los supervisores de los telecentros. Ver informe "Evaluación del Primer Curso Televisado para guías". Fondo de Capacitación Popular, Bogotá, mayo de 1971.

${ }^{31}$ Las razones de retiro del programa fueron el desgaste de las cintas, la presión de los usuarios y el consejo de los expertos consultados por la Dirección del Fondo (entre ellos estaba el Dr. Luis Antonio Bohórquez Casallas, quien adelantó una evaluación del curso de Alfabetización).
} 


\section{Los programas que se produjeron con carácter "provisional" no fueron tan provisionales y murieron viejos y desgastados.}

En el año de 1971, ante la urgencia de ofrecer "algo" a los usuarios en vista de que el curso de alfabetización había sido retirado de la programación, se dio inicio al diseño y producción de los cursos de post-alfabetización. Se elaboró un Nivel II equivalente a los cursos segundo y tercero de primaria y los llamados cuarto y quinto de educación básica.

Con esta programación en el aire se intentó hacer un cubrimiento total de las demandas de educación primaria de los adultos de Bogotá, Cundinamarca y Tolima.

De 1971 a 1973 se llevó a cabo una gran campaña de promoción del programa en las tres regiones antes citadas, logrando el mayor número de estudiantes inscritos durante toda la historia de Capacitación Popular ${ }^{32}$.

Para motivar la continuidad de los telecentros se experimentaron las más variadas estrategias. Lo más importante fue quizá vender la idea de que el telecentro era algo más que una simple tele-escuela. En el telecentro se podrían discutir todos los problemas de la comunidad y todo el mundo debería preocuparse por encontrar las soluciones a los problemas comunes. Los funcionarios de la Institución hablaban del telecentro como el "núcleo de la comunidad".

Por otra parte, el elemento básico en la organización y funcionamiento de los telecentros era el guía cultural. La Institución se dio a la tarea de capacitarlos y organizarlos. De esta forma no sólo se aspiraba a garantizar su permanencia en los telecentros por períodos largos, sino que era un mecanismo para suplir medianamente las deficiencias que presentaban los programas de televisión.

\section{Los estudiantes, en vez de aprender, olvidaban}

Aunque era de esperarse que la Institución estuviera en capacidad de producir programas de calidad, dado que trabajaban funcionarios con años de experiencia y habían mejorado momentáneamente los recursos financieros, no fue así. Una investigación acerca de los resultados de los cursos nuevos señalaba fallas protuberantes. Según los datos de una prueba de rendimiento básico académico aplicada a los estudiantes de uno de los cursos, no solamente no se habían presentado cambios en los conocimientos de los usuarios que habían estado expuestos al programa sino que habían olvidado lo que sabían antes de entrar al telecentro. Los estudiantes registraron mejores puntajes en el pre-test ${ }^{33}$ - Datos similares del rendimiento académico del curso cuarto fueron encontrados por otro estudio mucho más sofisticado ${ }^{34}$.

Para suavizar las críticas por la baja calidad de los programas, Capacitación Popular decidió darles un carácter de "Provisionales" a los cursos. Es decir, que estarían en el aire solamente mientras se diseñaban y producían otros cursos, esta vez si con todas las de la ley; pero esto tampoco fue así.

\footnotetext{
${ }^{32}$ En 1969 se organizaron 50 telecentros en Bogotá; en 1970 funcionaron 240 y en 1973 la suma llegó a 588, en Cundinamarca, Tolima y Antioquia. Debido al desgaste del Programa y a la falta de continuidad y compromiso por parte de los gobiernos con el mismo, en 1983 solamente quedan 70 telecentros y el programa se ha circunscrito, como al principio solamente a la ciudad de Bogotá; las demás zonas han sido abandonadas.

${ }^{33}$ Fondo de Capacitación Popular: "Investigación acerca del rendimiento del nivel II de educación básica para Adultos por TV". Noviembre 1971.

${ }^{34}$ Fondo de Capacitación Popular: "Evaluación del curso IV de Educación Básica”. Septiembre de 1976.
} 
Estos cursos provisionales estuvieron en la programación del canal hasta que las cintas de video-tape se deterioraron y se hizo su retiro y su archivo. El curso quinto se renovó en 1979 por otro quinto también provisional. El nivel II se retiró en 1977 y no se remplazó por ningún otro programa mientras que el curso cuarto fue remendado y después de diez años aparece todavía en programación.

\section{EI SENA por TV: Cómo aprender a planchar un pantalón y que le queden los prenses bien marcados}

Aparte de los programas para la enseñanza de los números y las letras, la presidencia de la República enfatizaba en que otra de las aspiraciones del proyecto era precisamente la de procurar la capacitación y el adiestramiento de los sectores populares en oficios semicalificados y técnicos. Por esto el nombre de "Capacitación"35.

La función de capacitar mano de obra correspondía al SENA, pero como esta entidad exigía una escolaridad mínima de quinto de primaria, una buena parte de los sectores populares quedaba por fuera del derecho a ser capacitados. En consecuencia el papel de la Institución aparecía claro y lógico: escolarizar estos sectores marginados para que pudieran ingresar al SENA y dieran comienzo a su adiestramiento técnico.

Esta función de Capacitación Popular en consonancia racional con otras entidades del Estado armonizaba además con la política del gobierno de Lleras de crear condiciones que sirvieran de base para impulsar la industrialización del país ${ }^{36}$.

La escolaridad de los sectores populares era además una necesidad sentida e impulsada por diferentes sectores sociales y políticos desde el comienzo de la década del setenta. Un ex-ministro de Educación aseguraba que "la industria no podía aportar tecnología avanzada, dada la imposibilidad del personal para captar mecanismos o innovaciones complejas. Las altas tasas de analfabetismo restringen y frenan la actividad económica del país"37.

Por otra parte el gobierno era consciente de la urgencia real de preparar y especialmente "disciplinar" adecuadamente la gran masa de campesinos que en la ciudad formaban el ejército de reserva para la industria y los servicios. De no utilizarse racionalmente estos recursos humanos podrían generar situaciones sociales y políticas cada vez más complicadas y difíciles de manejar.

Pero esta urgencia de capacitar los recursos humanos fue entendida por Capacitación Popular en una forma ambiciosa y desproporcionada. Pretendían no solamente escolarizar para que el SENA capacitara laboral y técnicamente sino que además emprendieron la aventura de crear un SENA por televisión. Lo que resultó fue una caricatura.

El proyecto era poder ofrecer a los adultos de los telecentros un nivel primario de capacitación en oficios varios. Con este propósito se inició en marzo de 1971 la producción y emisión de un programa denominado "Oficios semi-calificados".

\footnotetext{
${ }^{35}$ Una política similar parece ser la que busca desarrollar el programa del presidente Betancur quien también ha recalcado la necesidad de integrar al SENA a su programa de Educación a distancia. Ver: EL TIEMPO. 18 de septiembre de 1982. en que se comenta la inauguración de la Campaña de Instrucción Nacional - CAMINA-.

${ }^{36}$ Una exposición detallada de la política de industrialización del gobierno del Dr. Lleras puede encontrarse en Bejarano Jesús A.: Industrialización y politica económica (1950-19 76) Colombia Hoy. Bogotá, Edit. Siglo XXI. 1978.

${ }^{37}$ Mango, Daniel: “Informe del Ministerio de Educación Nacional al Congreso Nacional”. Bogotá, Imprenta del MEN, 1966. Pág. 75. 
El programa empezó unas 50 emisiones y los temas más relevantes eran los siguientes: Cómo reparar elementos ordinarios de la casa como: planchas, conexiones eléctricas, taburetes, mesas, ollas; cómo elaborar artefactos sencillos con materiales de desecho; cómo lavar y despercudir limpiones y hasta cómo planchar un pantalón para que le quedaran los prenses bien marcados.

Este programa fue suspendido por no reportar una audiencia en los telecentros que justificara la emisión.

Por lo menos dos razones ayudaban a explicar este fenómeno de no audiencia. En primer lugar aunque los oficios fueran de carácter elemental, se requerían para su aprendizaje algunos materiales que los estudiantes deberían llevar al telecentro para realizar las prácticas concretas. Los estudiantes jamás llevaron nada, dadas las características de pobreza generalizada.

La segunda razón era aún más irónica. Como los .estudiantes no llevaban los materiales para realizar las prácticas, la emisión de televisión se convertía en un recetario que indudablemente los usuarios no podían memorizar. La solución era acompañar la emisión televisada de algún material impreso que los usuarios pudieran coleccionar. Todo supuestamente funcionó hasta cuando descubrieron que los estudiantes no sabían leer.

Como vemos, estos tres programas que constituyeron el eje de la política de Capacitación Popular y las realizaciones prácticas y concretas del proyecto no fueron más que respuestas improvisadas y apresuradas a las exigencias políticas del momento. Todo esto a la larga, sólo podía conducir al fracaso y a una gran frustración por parte de los funcionarios que trabajaban dedicadamente a la elaboración y desarrollo de estos programas, así como de las personas a las cuales se dirigían las emisiones de televisión.

\section{Los Intelectuales en Capacitación Popular. Investigaciones, innovaciones educativas y frustraciones.}

Desde su fundación, el programa de Capacitación Popular se consideró novedoso. La investigación era central para su desarrollo. El programa sostenía ser el producto de importantes investigaciones y las transmisiones eran a su vez la consecuencia de estudios realizados por expertos en educación y comunicación. A la hora de la verdad no siempre ha sido así.

En los primeros años de vida de la Institución sorprendía el inusitado trajín de los investigadores. Un grupo de voluntarios suecos preparaba un informe evaluativo sobre el programa de Julio y Rosa; la sección de investigaciones adelantaba diferentes estudios sobre la recepción del programa; los usuarios, los guías, las actitudes y valores de la comunidad hacia el programa, etc., etc., ${ }^{38}$.

\footnotetext{
${ }^{38}$ Se hicieron investigaciones básicas para el programa de Alfabetización; investigación inicial y pre-encuestas para el nivel cívicosocial; pre-encuesta para el nivel de oficios; grupo de evaluaciones sucesivas tanto para las pruebas directas como para los circuitos cerrados y el primer ciclo en circuito abierto para el nivel de educación básica; una investigación sobre ahorro, de carácter nacional costeada por el Banco Popular; una investigación sobre recepción del canal 9 en Cundinamarca y Tolima; una investigación de casos con la colaboración de la Universidad Jorge Tadeo Lozano (Ver: Pedro Arreaza, "Informe sobre las actividades..." 1.c. p. 5-6). Además, se realizaron investigaciones sobre "Educación y Marginalidad". Bogotá, julio de 1972; "Quién es Julio y Quién es Rosa". Bogotá, noviembre de 1970. "Evaluación del Primer curso televisado para Guías". Bogotá, mayo de 1971. "Evaluación del Nivel II de Educación Básica”. Bogotá. noviembre de 1971.
} 
Como hemos dicho, Capacitación Popular había sido uno de los pocos programas que habían adquirido forma a partir de numerosos estudios previos. Hay constancia escrita de que se realizaron investigaciones sico-lingüísticas para conocer el índice de interpretación y memorización de imágenes, el nivel de significación de vocabulario y la facilidad de expresión oral de la población adulta del país.

Por medio de investigaciones socio-culturales se intentó conocer los intereses, las aspiraciones y los valores de los posibles usuarios de los programas frente a la educación, la recreación y la música. En general la Institución supuestamente había encontrado en los datos de la investigación un soporte sobre el cual construyó los programas, los cuales fueron posteriormente experimentados y evaluados en grupos de adultos de los barrios marginados ${ }^{39}$.

Daba la impresión de que todo en Capacitación Popular estaba mediado por el concepto de los investigadores. Efectivamente mucho de la seriedad y solemnidad que tenía el programa se fundamentaba en el carácter científico de las investigaciones que la Institución adelantaba. Con esta imagen se siguen empacando hasta hoy día los programas y los directivos no dejan de proclamar que todas las decisiones administrativas y pedagógicas están basadas en las conclusiones y las recomendaciones de los investigadores.

Hay que destacar que durante toda la administración del Dr. René Hauzeur, el segundo director de Capacitación Popular, los investigadores tuvieron todo el apoyo necesario para llevar adelante su trabajo - Producto de este esfuerzo combinado de técnicos y científicos fue indudablemente el "Modelo de Educación a Distancia", que aunque no llegó a ser más que una propuesta, constituyó un intento novedoso por implementar una política seria y responsable sobre el manejo de los medios masivos al servicio de la educación popular, y de conjugar el proceso administrativo con el investigativo.

Sin embargo, esta imagen del trabajo armónico y coherente entre políticos, ejecutivos e intelectuales muy pronto iba a comenzar a diluirse y a mostrar su verdadera cara. Pese a los intentos, algunos muy serios, por unir los dos procesos de administración e investigación, la verdad es que cada uno anduvo por su lado desconociéndose mutuamente.

\section{La investigación y los expertos de la Fundación Ford}

Prácticamente desde que se inició Capacitación Popular hasta mediados de la década del 70 la Fundación Ford estuvo vinculada a la sección de investigaciones y evaluación. Entre 1970 y 1971 donó cerca de un millón de pesos colombianos con el destino específico de organizar en mejor forma el departamento de investigaciones. Sugirió igualmente la formulación de proyectos para entrenar los futuros investigadores para lo cual se contaría con el apoyo financiero de la Fundación.

Para complementar estas acciones la Fundación contrató y envió como asesores a un grupo de "expertos" entre los cuales podríamos citar a las Dras. Rose K. Goldsen y

\footnotetext{
${ }^{39}$ Fondo de Capacitación Popular. "Memorias del Fondo de Capacitación Popular, 1967-1981”. Bogotá, abril de 1981. 
Regina E. Gibaja ${ }^{40}$.

Por lo menos dos hechos justificaban la participación de Ford en el proyecto de Capacitación Popular. En primer lugar fue una de las pocas entidades que respondió al llamado de ayuda y solidaridad que hizo Capacitación Popular a las más diversas organizaciones públicas y privadas, nacionales y extranjeras como una de las estrategias para lograr recursos financieros.

Por otra parte durante la década del 60 y comienzos del 70 gran parte de los recursos que las instituciones internacionales destinaban para la asistencia técnica de los países latinoamericanos se dedicaba a financiar y asesorar los proyectos educativos de cada región ${ }^{41}$. Por esta época también la televisión educativa fue considerada un avance tecnológico de los más importantes para solucionar los problemas educacionales masivos en América Latina ${ }^{42}$.

Durante esta primera etapa de trabajo de los expertos, es decir de 1970 a 1972, la sección de investigaciones adelantó variados proyectos de investigación y evaluación. Quizá el más importante fue el estudio de "Educación y Marginalidad" con el cual se intentó cubrir amplios aspectos de la realidad socio-económica de los usuarios, no sólo reales sino potenciales, del programa de Capacitación Popular ${ }^{43}$.

Aunque se hablaba de importantes hallazgos sobre motivaciones, actitudes e incluso situaciones políticas de "los marginados", este trabajo no aumentó ninguna de las decisiones administrativas que se tomaron en la época. El estudio que se tradujo al inglés y al francés, no fue difundido en el país.

Otros proyectos menos ambiciosos que el de "Educación y Marginalidad", se relacionaron más directamente con la Institución. Con estos trabajos se analizó el rendimiento académico de los usuarios del Nivel II, el impacto de los contenidos del programa de Julio y Rosa y se estudiaron más en detalle las situaciones de funcionamiento de los telecentros y las características de los usuarios del programa y de los guías culturales.

En resumen la venida de los expertos significó un mayor status para la sección de investigaciones pero no contribuyó en nada al mejoramiento del programa. La investigación siguió siendo un $1^{5}$ roceso aislado de las decisiones institucionales ${ }^{44}$.

\footnotetext{
${ }^{40}$ Quizá la persona más importante de la Fundación Ford que trabajó en Capacitación Popular fue la Doctora Regina E. Gibaja. Sus aportes tanto en la investigación como en la producción de los programas y en la recepción de los mismos delineaban una alternativa novedosa de utilizar los medios de comunicación en la educación. Desafortunadamente, los directivos no entendieron sus planteamientos y no establecieron los mecanismos de su aplicación.

La Dra. Gibaja produjo una serie de documentos importantes: "Televisión Educativa: Un enfoque para su evaluación". Bogotá, Fondo de Capacitación Popular, 1973. "Investigación en Televisión Educativa", en Revista del Centro de Estudios Educativos (México). Vol. IV, No. 2, 1974, p. 57-74. ¿"Es posible educar por televisión? Reflexiones sobre la educación de adultos por medios de comunicación de masas", en Revista del Centro de Estudios Educativos (México, No. 3), 1975.

${ }^{41}$ TIFFLIN, John: Problemas críticos en sistemas de televisión educativa” en Revista de Tecnología Educativa, Vol. 4 No. 2 , 1978.

${ }^{42}$ Hay que advertir que esta asistencia técnica y financiera de las entidades internacionales fue duramente cuestionada, especialmente por el movimiento estudiantil de 1971. (Ver: Lebot, Ivón: Educación e ideología en Colombia. Medellín: Ed. La Carreta, 1979, pág. 109).

${ }^{43}$ Este estudio fue realizado por un equipo mixto de Capacitación Popular y la Fundación Ford. Por parte de esta Fundación, que dirigía el proyecto, estuvieron: Marcial Antonio Riquelme, Rose K. Goldsen y Robert Arnove. Posteriormente se extraviaron los informes finales y solamente quedaron algunos borradores.

${ }^{44}$ En el caso de Capacitación Popular se aplica la tesis de Salan que dice: "La historia de la educación es una fuente inagotable de ejemplos acerca de la resistencia de las instituciones educativas a aceptar innovaciones". SOLARI, Aldo. "Desarrollo y política educacional en América Latina, en Revista de la CEPAL. Santiago de Chile. Primer semestre de 1977, p. 82.
} 


\section{El desgaste de una institución}

Hemos visto, cómo el Fondo de Capacitación Popular Le una institución que se creó por lo alto. Dependía de la Presidencia de la República, ya que un consejero presidencial había sido su fundador y principal ideólogo.

Eh Fondo debería estar dirigido por un Consejo Directivo compuesto por: el Consejero Presidencial, el Director y dos delegados de la Junta Directiva de Inravisión y por los Ministros de Educación, Comunicaciones y Gobierno.

Formal y aparentemente el Estado le había proporcionado su respaldo al Fondo. En realidad no todo funcionó como debía. Por ejemplo, el Consejo Directivo sólo se reunió el día de la inauguración y no alcanzó a conocer siquiera los problemas por los que atravesaba Capacitación Popular en sus comienzos ante la insuficiencia de sus recursos financieros.

A pesar de que el Consejo Directivo no funcionó, el Fondo tuvo cierta respetabilidad en el ambiente oficial mientras dependió de la Presidencia y del consejero Galat. Con la salida de éste, al final del gobierno de Pastrana en 1974, el programa de Capacitación Popular pasó a depender del Instituto Colombiano de Radio y Televisión.

Es importante recalcar, que, el programa de Capacitación Popular fue fundado bajo la presidencia de Carlos Lleras Restrepo y durante su administración hubo un gran interés por eh mismo.

Durante el gobierno de Misael Pastrana Borrero, el entusiasmo continuó y la institución logró su máximo auge. Pero a medida que iban avanzando los gobiernos, el interés por Capacitación Popular iba disminuyendo.

Es básico insistir en el papel que jugó eh consejero Galat. Su permanencia en el gobierno durante la administración tanto de Carlos Lleras Res-trepo como el gobierno de Pastrana Borrero explican el por qué el programa tuvo cierta continuidad.

Es importante recalcar un hecho que no es atípico de la administración pública colombiana y es que la dependencia del programa de su gestor fue tan grande que su vida, en gran parte, dependió de él, y a su vez su salida significó el comienzo de su inanición.

Paradójicamente lo que ayer era parte importante del proyecto nacional de "Integración Popular" hoy es tan sólo una "rueda suelta" en el contexto de la administración pública. Los gobiernos de López Michelsen y de Turbay Ayala no le prestaron mayor atención a Capacitación Popular. El Ministerio de Educación se contentó con hacer algunos aportes. INRAVISION se limitó a administrar los recursos financieros asignados por el Ministerio de Educación Nacional ${ }^{45}$.

\footnotetext{
${ }^{45}$ Entre otras dificultades está la de no haberle conferido, una legalidad al Fondo, anexo a Inravisión. En los primeros días era manejado desde la Presidencia de la República. Luego desde el Ministerio de Educación Nacional; a ratos era autónomo y a ratos no. Presupuestalmente ha sobrevivido mediante un convenio entre Inravisión y el Ministerio de Educación renovado año tras año desde 1969. Los aportes en la actualidad no alcanzan para el funcionamiento. Los usuarios de los programas deben subvencionar los gastos institucionales mediante el pago de los materiales impresos que vende la entidad a través de la Caja Agraria.
} 


\section{¿En qué terminó la programación de Capacitación Popular?}

Ante la falta de compromisos por parte de los últimos gobiernos y la incapacidad para continuar con unos programas, pero enfrentados al hecho concreto de unas horas de televisión que tienen que llenar, se hizo lo de siempre: invitar a las embajadas de los países amigos para que fueran ellas las que con películas llenaran los espacios del canal $^{46}$.

Quizá la única alternativa, debido a la falta de recursos técnicos y humanos para producir televisión, fue acudir a las cinematecas de las embajadas. Era la única parte en donde se encontraba material de calidad aceptable y a ningún costo.

Las administraciones, especialmente después de 1976, recurrieron a esta alternativa fácil para proporcionarle una programación al canal. A partir de este año la programación de las embajadas fue aumentando paulatinamente. Los directores del Fondo se dedicaron de ahora en adelante a conseguir unos fondos para mantener una burocracia y a llevar unas relaciones cordiales con las embajadas que se convirtieron en proveedores de materiales para el canal.

No es por azar que se inició un programa llamado "Días Nacionales" dedicado a celebrar el Día Nacional de los países con los cuales Colombia mantenía relaciones diplomáticas y comerciales. En este programa, que se mantiene hasta hoy en día, participa algún representante diplomático del país que celebra su día patrio y conmemoran diversos aspectos culturales, turísticos o de intercambio comercial que sean de interés para los colombianos.

Todo esto ha hecho que entre la institución y las embajadas se establezcan las más estrechas y amistosas relaciones de interés mutuo. A Capacitación le interesa conservar a su disposición una gran cinemateca y a las embajadas de los países amigos un canal de televisión convertido en una vitrina gratuita para divulgar los más variados temas sobre sus respectivos países. En pocas palabras, Capacitación Popular ha convertido a las embajadas en programadoras del Canal 11.

\section{Ante la improvisación y descuido, se infiltra el "fantasma" del comunismo}

Siempre que la Institución se refiere a la participación de las embajadas en programación del canal educativo habla de "Los países amigos" sin hacer ninguna diferenciación ${ }^{47}$.

En este reducido grupo de "países amigos" se encuentran los Estados Unidos, Alemania Federal, Francia, Italia y otros países de Europa Occidental. De los demás países sólo se hace referencia el día que celebran su Día Nacionall ${ }^{48}$.

Suponemos que esto se deba a que son muy pocos los países que disponen de recursos para la producción de este tipo de materiales o porque si los producen no tienen el interés suficiente de que sean puestos en la programación del canal, o simplemente porque no los envían.

\footnotetext{
${ }^{46}$ Ver: Capítulo II "Las Licitaciones”, en Azriel Bibliowicz. Lo público es privado: un análisis de la televisión colombiana. Ithaca, New York, Dissertation Series, Cornell University. January, 1979.

${ }^{47}$ Informes de programación de la Sección de Producción Educativa del Fondo de Capacitación Popular. Igualmente, Fondo de Capacitación Popular, "Memorias del Fondo de Capacitación Popular, 1967-1981". Bogotá, abril de 1981.

${ }^{48}$ Ibídem.
} 
Sin embargo, sabemos que un comentario de la editorial del periódico "El Tiempo" causó dificultades para la Institución y se generó una especie de censura a los materiales fílmicos que provenían de los países socialistas. El comentarista afirmó que consciente o inconscientemente el Canal Educativo se estaba convirtiendo en una especie de vitrina que divulgaba "ideas extrañas". Se refería a una película que provenía de Polonia.

Por supuesto que este comentario del periódico El Tiempo ${ }^{49}$ hizo hablar de purgas y despidos en Capacitación Popular. Pero cuando fueron a examinar el programa con detenimiento sólo se trataba de una película de artesanías en miniatura de los campesinos polacos. No había nada de extraño ni se estaba haciendo ningún tipo de proselitismo.

En el segundo semestre de 1981 ante el inminente riesgo de tener que repetir enlatados, se regresó a las cinematecas de los países socialistas, ya que indiscutiblemente son ellos quienes hacen los mejores documentales artísticos, científicos y turísticos. Obviamente se tiene el mayor de los cuidados en no dejar infiltrar algunas de esas "ideas extrañas", que no son compatibles con las que, de acuerdo con el gobierno, deben tener los colombianos.

\section{Capacitación Popular hoy: Un caso típico del manejo burocrático del Estado.}

Cuando hacíamos la reconstrucción de los datos sobre la historia de Capacitación Popular $^{50}$ nos dábamos cuenta no sólo de la deficiencia de los resultados de un proyecto educativo y del manejo inadecuado de los medios masivos. Observábamos también que el estilo con que los directivos manejan en la actualidad no es un estilo diferente al que se utiliza en el conjunto de las entidades del Estado ${ }^{51}$.

Al Estado colombiano no parece preocuparle la suerte que corran las entidades que él mismo crea. No se encuentra ninguna razón lógica para que primero se haya establecido Capacitación Popular y luego se deje a la deriva sin señalarle unos objetivos concretos.

En la administración pública todo parece indicar que al final se consolida el clientelismo. Este sistema de administración basado en relaciones de amistad y compadrazgo desplazan la profesionalidad y eficacia de los cuadros de una organización burocrática racional $^{52}$.

La burocracia estatal es una realidad con una dinámica propia que va más allá de los individuos que la componen ${ }^{53}$. Cuando el proceso burocrático se apoderó del programa

\footnotetext{
${ }^{49}$ Ver: EL TIEMPO, Bogotá, lunes 22 de junio de 1981.

${ }^{50}$ Seis administraciones han pasado por Capacitación Popular: Pedro Arreaza Lleras (1967-1969), René Hauzeur Forero (19691974), Pedro Arreaza Lleras (1974-1977), Fernando Alford Cortázar (1977-1979), Magaly Rosales de Gómez (1980-1982) y Diego Castillo Sánchez (1982...).

${ }^{51}$ Las burocracias, afirma Caplow, "nunca obedecen exactamente los textos de sus estatutos, están sujetas a numerosas desviaciones de función y de estilo, y pueden adquirir características completamente imprevistas" (Theodore Caplow, op. cit. p. 111). Este es el caso de las administraciones en Capacitación Popular.

${ }^{52}$ Max Weber reaccionó airadamente contra este fenómeno en el cual veía una progresiva limitación de la conciencia humana y una reducción de la libertad individual. "Es horrible pensar que el mundo pudiera algún día poblarse de nada más que de pequeñas ruedas dentadas, de hombrecitos asidos a pequeños puestos esforzándose sólo por alcanzar otros de mayor importancia”. Citado por Gonzalo Cataño, "Nueva lectura de Max Weber", en Revista ECO. Bogotá, abril de 1980. No. 222. p. 621.

${ }^{53}$ Ver: MERTON, Robert K. "Frustraciones del intelectual en la Burocracia", en Teoría y Estructura Sociales. México: Fondo de Cultura Económica, 1980. p. 302-304.
} 
fue paulatinamente sustituyendo a los investigadores y sacándolos de los niveles decisorios de la Institución ${ }^{54}$.

\section{La frustración y el cansancio}

Como habíamos explicado, al comienzo existía un gran entusiasmo por el programa.

De 1970 a 1973, en Capacitación Popular era contagioso el empeño que se ponía en el desarrollo de cualquier labor. A algunos de los funcionarios más dedicados se les llamó "apóstoles de la comunidad" y en general los visitantes se quedaban sorprendidos de encontrar caras nuevas y deseosas de prestar un servicio de la mejor forma posible. En aquellos días, no pocas veces se escuchó el comentario que Capacitación Popular no parecía una entidad del Estado.

Pero con el tiempo, el desgaste de unos programas con la falta de compromiso de los últimos gobiernos y con la llegada de la burocracia cliente-lista, el empeño por el trabajo pronto se convirtió en frustración ${ }^{55}$.

Todo lo que se había hecho con tanto esfuerzo ya no servía a los administradores y era considerado peligroso. Un ejemplo de esto sería la persecución de la cual fueron objeto los guías de los telecentros y los coordinadores de los barrios populares ${ }^{56}$.

Paulatinamente se incrementó el papeleo, los trámites, etc., y todo lo que es típico de una organización cansada y parsimoniosa.

Si analizamos la Institución actualmente encontramos que es una empresa con dificultades tanto financieras como administrativas. No existe ningún compromiso por parte del Estado y está destinada a morir por inanición. Este es el destino más probable si se tiene en cuenta el congelamiento de sus recursos financieros, la incapacidad de las directivas para buscar otras fuentes de financiación y el silencio profundo y cómplice de las diferentes instituciones y personas que de una u otra forma son responsables del funcionamiento del programa.

Un indicador de la indefinición por parte del Estado en cuanto a lo que es y debe ser Capacitación Popular fue la solicitud de Gloria Zea de Uribe, durante varios meses, para que le adjudicaran el Canal ${ }^{57}$. La señora Zea quería transformar el Canal en un canal cultural. Ya por esta época el canal no se veía como exclusivamente educativo y los programas, aun cuando sus funcionarios no los aceptaran como un fracaso, eran considerados así por otras entidades del Estado.

\footnotetext{
${ }^{54}$ Estudios como el de Michel Crozier (El fenómeno Burocrático) han mostrado que "la organización de los sistemas burocráticos puede variar hasta el infinito y que, por otra parte, ninguna funciona según el plan de sus creadores. En algunos aspectos, los objetivos que marcaron la creación de una burocracia son irreconocibles a causa de los cambios introducidos por los funcionarios en razón de sus intereses personales". (CAPLOW, Theodore. La Investigación Sociológica. Barcelona: Editorial Laia, 1977, p. $114)$.

${ }^{55}$ Gouldner mostró cómo y por qué el cambio de una persona en un puesto directivo podía llevar consigo la posibilidad de determinar cambios en el estilo administrativo, en el sistema de normas y en la jerarquía de la organización. Alvin W. Gouldner. Patterns of indos trial bureaucrac3. Glencoe, Illinois: The Free Press, 1954.

${ }^{56}$ En septiembre de 1976, Pedro Arreaza que fue también el tercer Director del Fondo, mediante una carta, prohibió a los coordinadores de los telecentros que tuvieran cualquier contacto con los telecentros y las organizaciones de la comunidad. Esta orden se mantuvo hasta febrero de 1977.

57 El momento en que la directora de la entidad estatal encargada de orientar la cultura en el país, el Instituto Colombiano de Cultura - COLCULTURA, hizo la solicitud fue cuando tuvo mayor apoyo gubernamental y cuando su padre se desempeñaba como Ministro de Gobierno. A pesar de ello, nunca pudo lograr su propósito.
} 
Otro hecho que curiosamente indica la gran indefinición respecto de Capacitación Popular es el constante cambio de logos para su propia identificación ${ }^{58}$. Es como si se creyera que el cambio de logo revitaliza o les cambia la imagen y el estado de deterioro de los programas.

\section{Una burocracia que no comprende su realidad}

Es interesante anotar que uno de los problemas de Capacitación Popular es que ella misma, en otras palabras sus funcionarios, no reconocen los problemas internos. La burocracia que la maneja se ha anquilosado y tiene miedo de aceptar los problemas que aquejan a la Institución. No sería atrevido afirmar que ya hasta tienen un comportamiento cínico sobre el programa que administran y ejecutan ${ }^{59}$.

En entrevistas conferidas a los medios de comunicación sobre la institución, no hay una posición clara sobre el particular ${ }^{60}$. Es como si aceptar que los problemas que aquejan a la Institución y la falta de compromiso que tiene el gobierno con la misma significara la firma del burócrata de su propia sentencia ${ }^{61}$.

\section{¿Un nuevo programa de Educación a Distancia?}

Es interesante analizar el desarrollo de los programas de Betancur a la luz de la experiencia de Capacitación Popular.

Como hemos visto a través de este artículo, no existe ninguna garantía de que un programa que se inicia en un gobierno sea continuado por otro. Más aún, debido a que un programa fue iniciado por una administración, la que sigue, probablemente, lo va a acabar o no le prestará la debida atención por cuanto no lo identifica como suyo. Este problema de la falta de continuidad y planeación ha sido uno de los pecados permanentes de la administración pública colombiana.

La falta de continuidad de los programas parece ser una característica estructural de los planes de gobierno y de la burocracia estatal. Por ello el caso de Capacitación Popular parece no ser atípico. Ante esto nos preguntamos: ¿Cuál será el futuro de los programas educativos que emprende actualmente el presidente Betancur?

\footnotetext{
${ }^{58}$ El primer logo fue "Capacitación Popular: Palanca del Progreso", que duró hasta 1974, en que fue remplazado por "TV-11". Luego se le agregó "TV 11, Más Televisión”, con una pretensión de competir con los canales comerciales. En 1977 se introdujo el logo "Televisión Educativa y Cultural", igual al que Inravisión tiene para identificar su programación dirigida a las escuelas públicas. En 1978 se modificó el logo como "Televisión Cultural" que permanece hasta ahora.

59 Goffman llama "cínico" al individuo que no deposita confianza en sus actos ni le interesan mayormente las creencias de su público; a aquel que puede experimentar una especie de gozosa agresión espiritual ante la posibilidad de jugar a voluntad con algo que su público puede tomar seriamente. GOFFMAN, Erving. La Presentación de la persona en la vida cotidiana. Buenos Aires: Amorrortu Editores, 1971, p. 29-30.

${ }^{60}$ Sobre el particular se puede ver: Azriel Bibliowicz, “¿Canal Educativo o Cultural?”, en Tele-Revista de EL ESPECTADOR. Bogotá, 19 de abril de 1980. No. 56, p. 12-13. Azriel Bibliowicz, "Los enlatados más gastados y aburridos de la televisión colombiana", en Tele-Revista de EL ESPECTADOR. Bogotá, 20 de marzo de 1982. p. 12-13. Azriel Bibliowicz, "La muerte de un Canal", en Tele-Revista de EL ESPECTADOR. Bogotá, 13 de diciembre de 1980. p. 12-13. Castro Caycedo, Gustavo, "Gloria pide un canal de televisión al Gobierno", en EL TIEMPO. Bogotá, 21 de diciembre de 1980. Castro Caycedo, Gustavo. "Gloria insiste en el canal de Televisión" en Revista Elenco de EL TIEMPO. Bogotá, 19 de marzo de 1981.

${ }^{61}$ Esta situación contrasta con los planteamientos de Merton acerca de lo que debe ser la burocracia. "El mérito principal, sostiene Merton. de la burocracia es su eficacia técnica, con una gran estimación por la precisión, la rapidez, el control experto, la continuidad, la discreción y la óptima restitución del gasto que representa. La estructura se aproxima a la eliminación completa de relaciones personalizadas y de consideraciones no racionales (hostilidad, ansiedad, complicaciones sentimentales, etc."), MERTON, Robert K. op. cit. p. 276.
} 
¿Qué garantías tiene este gobierno de que su programa no será remplazado por la próxima administración como ésta lo ha hecho respecto a la anterior ${ }^{62}$. Hasta dónde esta rotación permanente de programas educativos ha tenido como consecuencia el que el país no cuente con un verdadero plan educativo y el número de analfabetas sea cada vez mayor?

¿Cuáles son las garantías de que los programas educativos recibirán un financiamiento adecuado en éste y en los próximos gobiernos? ¿Cuáles son las garantías de que el aparato estatal no caerá en los pecados de la burocracia clientelista y anquilosada?

¿Qué garantías se ofrecen para que los programas no dependan exclusivamente de las voluntades de unas personas sino que tienen una continuidad institucional?

¿Cuáles son las garantías de que los alfabetizadores recibirán la preparación adecuada para el cumplimiento de su labor y de que serán estimulados?

Estos son sólo algunos de los interrogantes que surgen ante el análisis del Fondo de Capacitación Popular, un programa que arrancó con el impulso y el ánimo, que hemos visto desplegados para la campaña de Institución - Camina- en el Palacio de Nariño, casa de los Presidentes de Colombia ${ }^{63}$

\section{Unas reflexiones necesarias:}

Los planteamientos esbozados llevan a unas reflexiones finales sobre esta experiencia educativa a distancia, la primera y más importante en la educación de los adultos en el país:

- Los objetivos propuestos desbordaron cualquier capacidad imaginativa hasta el punto de creer que la Operación Capacitación Popular sería la tabla de salvación de los problemas educativos de la población adulta del país, pero también de los económicos, sociales y políticos en su conjunto.

La experiencia misma demostró lo idealista de los planteamientos y las limitadas capacidades para acercarse a ellos. La distancia demolió el entusiasmo inicial y causó frustración institucional, hasta el punto de que hoy día son muy pocos los funcionarios que están convencidos de lo que hacen. Los objetivos comunitarios se echaron por la borda y se suplieron por las necesidades individuales de la conservación del cargo.

Las buenas intenciones de los gestores no fueron suficientes para alimentar el entusiasmo por mucho tiempo. Tal vez constituyeron una condición necesaria cuando se inició la Operación Capacitación Popular. Pero cuando las bases de la Integración Popular se demolieron, también se derrumbó la consecuencia de ella que fue el programa mismo.

\footnotetext{
${ }^{62}$ La Campaña de Instrucción Nacional - CAMINA - del presidente Belisario Betancur, remplaza la Campaña Nacional de Alfabetización "Simón Bolívar" fundada bajo la presidencia de Julio César Turbay Ayala, en 1980.

${ }^{63}$ La inauguración de la Campaña de Instrucción Nacional - CAMINA - se hizo el viernes 17 de septiembre en la Casa de Nariño. El acto se realizó con la participación de personajes tanto del sector público como del privado y de artistas afamados. Para el acto se compuso un himno $y$ fue ampliamente difundido. INRAVISION dedicó una hora para transmitir el evento a todo el país, el sábado 18 de septiembre, de 6 a 7 p.m. por la primera cadena. (Ver: EL TIEMPO, 18 de septiembre de 1982>. EL ESPECTADOR, 18 de septiembre de 1982.
} 
El programa no pasó de ser un buen intento de acercamiento a la educación de los adultos por medios como la televisión. Y, como dicen Grobb y Lazerson, "los intentos de reformar las escuelas siempre se han incrementado en períodos de tensión social" ${ }^{4}$. La década de los sesenta fue especialmente rica en explosiones de protesta desde el campo, desde la industria, desde la educación, desde la política, desde la Iglesia. Todas las instituciones se removieron durante el período. $Y$ en este contexto conflictivo nace Capacitación Popular.

- Las estructuras sociales debieron salir al paso a los problemas. Pero un programa dirigido por las instituciones burguesas muy difícilmente pudo llegar a las estructuras populares. Se probó que cuanto más alejado está el grupo destinatario de los valores de quienes guían el programa, más improbable su asimilación por parte de los usuarios. Como dice Claude Grignon, "no es fácil para una institución fundada en los valores de la sociedad burguesa recuperar y reintegrar a los jóvenes formados en la asistencia a clases de tipo popular desde su edad más temprana" ${ }^{* 65}$, Este es el caso de Capacitación Popular y los usuarios "marginados" en un intento por conquistarlos y subsumirlos dentro de las estructuras del sistema. La institución no pudo ni supo comprender la lógica popular, a pesar de que siempre se planteó que el programa partía del conocimiento de las comunidades.

- La organización burocrática fue un Max Weber al revés: ausencia de racionalidad, disparidad de los medios respecto de los fines, falta de cálculo de las operaciones, no utilización del saber profesional, no establecimiento de niveles por capacidades.

Se partió del supuesto que la "hibridez institucional" sería una garantía. Pero ella llevó precisamente a su fracaso. Ni fue Presidencia de la República, ni fue Ministerio de Educación, ni fue Ministerio de Gobierno, ni fue Ministerio de Comunicaciones, ni fue INRAVISION, porque las dependencias del Estado se manejan como islas con escasos vasos comunicantes. La Integración Popular pretendió atacar y derrumbar el individualismo de los marginados analfabetas pero no el de las instituciones del Estado.

Existe, por otra parte, un maniqueísmo a favor del "ego institucional" expresado en que cada entidad se cree buena y las demás las malas, las ineficientes, las burocratizadas y las que deben ser corregidas. Y este es el caso de Capacitación Popular respecto de sí misma y de las demás entidades del Estado. Se advierte una añoranza por no haber podido ser otra cosa, por ejemplo una empresa privada en la que, supuestamente, hay eficiencia, rentabilidad y éxito. Son pocos los funcionarios que conocen la empresa privada en sus dimensiones interiores, ya que su carrera burocrática ha transitado por entidades del Estado.

En esa carrera se aprende la esquizofrenia institucional: hacia dentro los funcionarios pueden ser críticos, abiertos, y socializarse en la burocracia; pero hacia fuera deben ser "leales", hablar siempre en positivo y estar dispuestos a defender la entidad. Una doble cara, como plantea Goffman, es característica de un buen funcionario ${ }^{66}$, que debe aprender a ponerse y a usar la máscara.

\footnotetext{
${ }^{64}$ GRUBB, W. Norton y MARVIN Lazerson, "Continuidad y falacia en la educación profesional”, en BIASUTTO, Carlos. Educación y Clase Obrera. México, Editorial Nueva Imagen, 1978. p. 32.

${ }^{65}$ GRIGNON, Claude, “Cómo se fabrica un buen obrero o las funciones sociales de la enseñanza técnica?”, en GRAS, Alain. Sociología de la Educación. Textos Fundamentales. Madrid, Narcea Ediciones, 1976. p. 309.

${ }^{66}$ GOFFMAN, Erving. op. cit.
} 
- Como la Televisión Educativa, en general en sus primeras fases, el programa de Capacitación Popular "se desarrolló sobre el supuesto, muy cuestionable, de que la educación seguiría el mismo desarrollo histórico que la manufactura, de modo que los medios educativos vendrían a multiplicar la efectividad del trabajo humano en educación, sustituyendo, aunque fuera parcialmente, el trabajo de los educadores", como dice Mockus ${ }^{67}$. Los maestros, aunque se sostuvo lo contrario, fueron remplazados por estudiantes de bachillerato sin experiencia ni formación profesional-pedagógica ni de proyección comunitaria. Se asignó como consecuencia la mayor responsabilidad a la televisión, medio que se consideró como autosuficiente, capaz por sí mismo de "educar" a los adultos marginados. La innovatividad del programa enfatizaba en este poder casi ilimitado de la televisión. Cuando la entidad se dio cuenta del fracaso todo el proyecto se había derrumbado.

- La falta de continuidad del programa y del apoyo gubernamental es evidente tanto durante los dos gobiernos del Frente Nacional (Lleras Res-trepo y Pastrana Borrero) como en los tres siguientes. Sin embargo, cuando ha habido necesidad de popularidad se ha apelado a la mención de los logros institucionales. Como planteaba Arnove, el Frente Nacional, dentro del cual nació el programa, "fue admirablemente experto en crear la imagen de gobiernos malévolos y orientados al cambio, que promovían el desarrollo social mediante la extensión de los servicios educativos. Al final del Frente Nacional los sistemas elitistas existentes, el social y educativo en este caso, no sólo seguían intactos sino robustecidos, confirmando de esta manera el adagio francés: mientras más cambian las cosas más permanecen lo mismo" ${ }^{68}$.

En el caso de Capacitación Popular la crítica puede llegar a mayores profundidades: no solamente las cosas permanecieron lo mismo sino que retrocedieron. La tarea básica del programa es la educación en los niveles de alfabetización y educación básica de la población adulta del país. En 1967, cuando se inició el programa, se pensaba alfabetizar a dos millones de personas ${ }^{69}$. Hasta 1983 la suma de inscritos en el programa solamente llegaba a 152.000 estudiantes adultos. Esta cifra muestra un promedio semestral de inscritos inferior a 5.000 personas para un programa 'masivo, acelerado y barato" como se planteaba. A este promedio hay que descontar las deserciones de los usuarios durante el curso del programa.

Cuando el presidente Belisario Betancur visitó Nariño, al cumplirse el primer año de gobierno, afirmaba que "en alfabetización vamos de para atrás" ${ }^{\text {" }}$, y mostraba que hace treinta años los analfabetas eran el 400/o de la población (diez millones de habitantes), es decir cuatro millones de personas, y que hoy día son el 200/o (sobre 28 millones), es decir 5.6 millones, lo cual implica el retroceso.

Si se consiguiera adelantar una política de ampliación de la educación en los sectores populares y a bajo costo, sin que ello significara la calidad inferior, se habría dado uno de los pasos más importantes en lo que se ha denominado desde los años sesenta la "democratización" de la educación. Por razones de crecimiento vegetativo y de la extremada juventud de la población, el sólo hecho de mantener la situación deficitaria existente implica grandes esfuerzos, aún antes de pretender corregirla. Silos programas, como dice Ana M. de Babini, tienen éxito, "su papel es por definición

\footnotetext{
${ }^{67}$ MOCKUS, Antanas y otros. "La Reforma curricular y el Magisterio", en Tribuna Pedagógica. Bogotá., Asociación Distrital de Educadores (ADE), No. 1, 1982. p. 8-9.

${ }^{68}$ ARNOVE, Robert. op. cit. p. 44.

${ }^{69}$ ARREAZA LLERAS, Pedro. "Informe sobre las actividades...". 1. c.

${ }^{70}$ El Tiempo. Bogotá, 21 de agosto de 1983. p. 7-A. También: El Tiempo. Bogotá, 23 de agosto de 1983. p. 4-A.
} 
transitorio en su concepción actual; si fracasan, en cambio, su existencia puede estar asegurada por largo tiempo"71. ¿Cuál será la perspectiva de la educación de los adultos y de las entidades creadas para cumplir con esa tarea? Indudablemente, Capacitación Popular debe morir para renacer con otro estilo, con otras estrategias, con otras políticas, con otras metodologías, y con otros resultados.

\footnotetext{
${ }^{71}$ EICHELBAUM DE BABINI, Ana María, "Algunas ideas para la fundamentación sociológica del programa de Educación Básica". Bogotá, Fondo de Capacitación Popular, julio 14 de 1975. (Mecanografiado).
} 